\title{
HYDROGEN AND NITROGEN AMBIENT EFFECTS ON EPITAXIAL LATERAL OVERGROWTH (ELO) OF GaN VIA METALORGANIC VAPOR-PHASE EPITAXY (MOVPE)
}

\author{
Kazuyuki Tadatomo, Yoichiro Ohuchi, Hiroaki Okagawa, Hirotaka Itoh, \\ *Hideto Miyake and *Kazumasa Hiramatsu \\ Mitsubishi Cable Industries, Ltd., Central Research Laboratory, Japan \\ Hideto Miyake, Kazumasa Hiramatsu \\ * Dept. of Electrical \& Electronic Eng. Mie University, Japan
}

Ambient gas effect on the epitaxial lateral overgrowth (ELO) of GaN via metalorganic vapor-phase epitaxy (MOVPE) on a MOVPE-grown GaN (0001) / sapphire (0001) substrate with a $\mathrm{SiO}_{2}$ stripe mask has been studied by means of field-emission scanning electron microscopy (SEM) and highresolution X-ray diffraction (XRD) analysis. Different ambient gases of nitrogen, hydrogen and their mixture (mixture ratio, hydrogen : nitrogen $=1: 1$ ) affect the lateral overgrowth rate, the surface morphology and the crystalline tilting of ELO-GaN layers. XRD revealed that the ELO-GaN layer on the $\mathrm{SiO}_{2}$ mask aligned along the $\langle 1 \overline{1} 00\rangle$ direction exhibited anisotropic crystalline tilting toward $<11 \overline{2}$ $0>$. For ELO-GaN growth in nitrogen ambient, the growth rate of the (0001) facet decreases, the lateral overgrowth rate increases and the tilting of the ELO-GaN layer increases, while no smooth surface is obtained, in comparison with ELO-GaN growth in hydrogen ambient. For the mixture ambient, a smooth surface with a fast lateral overgrowth rate is achieved and the dislocation density is not more than $10^{7} \mathrm{~cm}^{-2}$, which is comparable to that in hydrogen ambient.

\section{Introduction}

Recently, high-performance optical devices such as light-emitting diodes (LEDs) and laser diodes (LDs) in green and ultraviolet regions have been developed using GaN, AlGaN and InGaN compound semiconductors $[1,2]$. These GaN-related devices are usually fabricated on a sapphire substrate, because there is no large-scale substrate for $\mathrm{GaN}$ heteroepitaxial growth. Due to the large differences in lattice constant, thermal expansion coefficient and chemical nature between $\mathrm{GaN}$ and sapphire, many threading dislocations are formed from the GaN or AlN buffer layer on the sapphire substrate, and the dislocation density has been reported to be of the order of $10^{9}-10^{10} \mathrm{~cm}^{-2}$ [3]. Although high-brightness LEDs have been realized in spite of the high dislocation density, the reduction of the dislocation density is desired to improve the performance and reliability of these devices. The epitaxial lateral overgrowth (ELO) technique based on the selective area growth (SAG) has recently attracted considerable attention, since the fabrication of blue-violet laser diodes on the ELO-GaN layer using this technique resulted in the achievement of a long lifetime of more than 10,000 hours [4].

The SAG in MOVPE on GaN/sapphire and $\mathrm{AlGaN} /$ sapphire with $\mathrm{SiO}_{2}$ stripe patterns was published for the first time in 1994 by Kato et al. [5], followed by that on GaN/sapphire [6]. Intensive studies on SAG have revealed that the ELO effectively reduces the dislocation density [4, 7-11]. The crystalline properties and the growth mechanism of ELO-GaN can be affected by mask patterns such as stripe direction [10-13] and growth conditions such as growth temperature [10], source gases [10], growth pressure and ambient gas. However, the effects of ambient gas remain unclear. We investigate the effects of hydrogen gas, nitrogen gas and their mixture in an atmospheric MOVPE on ELO-GaN. 

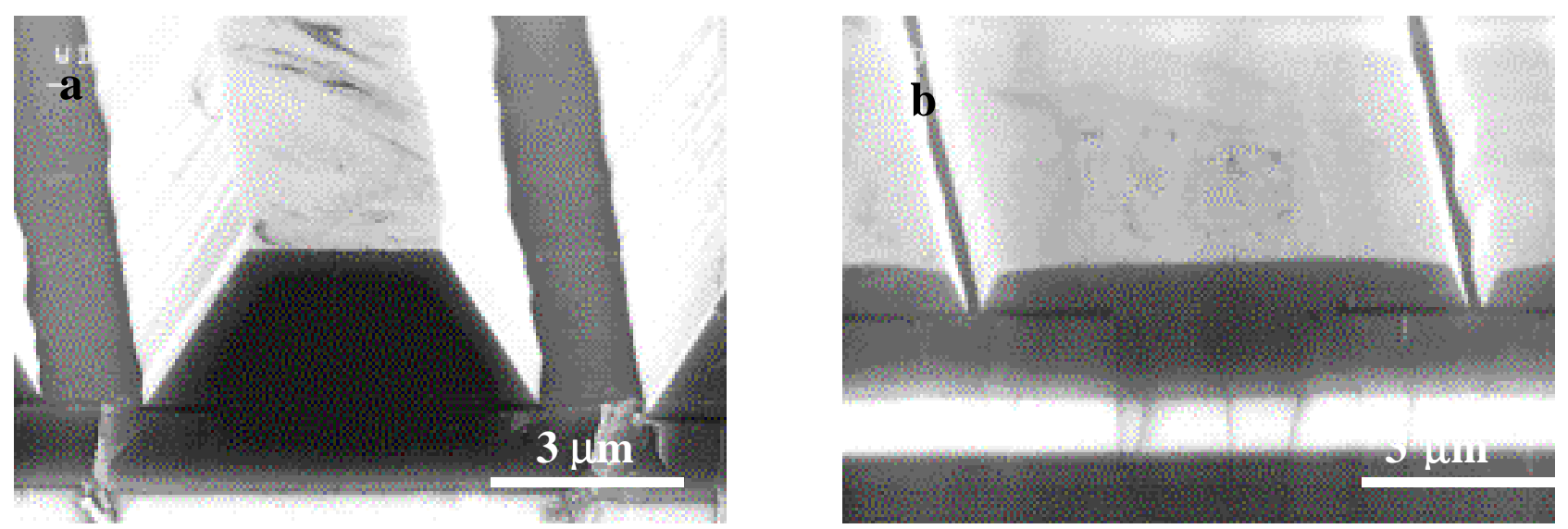

Figure 1 SEM images of $\mathrm{GaN}$ on $\mathrm{SiO}_{2}$ stripe pattern along the $<1 \overline{1} 00>$ direction at a growth time of 30 min in (a) hydrogen ambient and (b) nitrogen ambient.
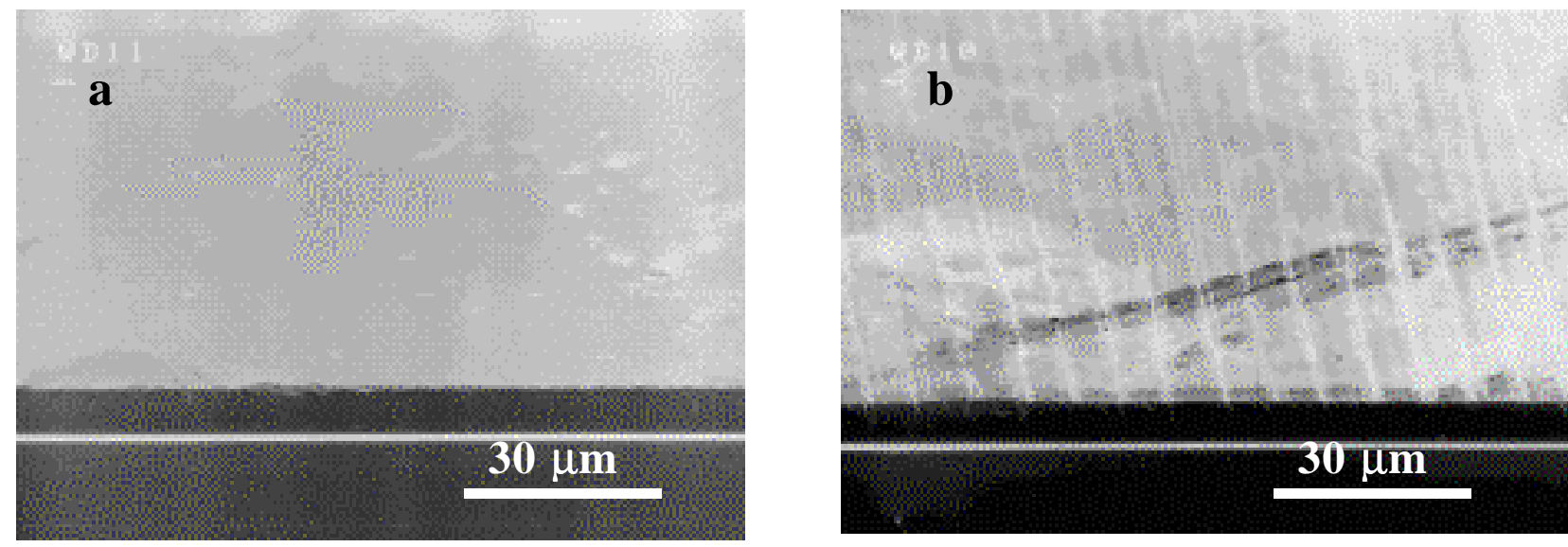

Figure $2 \mathrm{SEM}$ images of $\mathrm{GaN}$ layer grown by ELO on $\mathrm{SiO}_{2}$ stripe pattern along the $\langle 1 \overline{1} 00\rangle$ direction (a) at a growth time of $120 \mathrm{~min}$ in hydrogen ambient and (b) at a growth time of $180 \mathrm{~min}$ in nitrogen ambient.
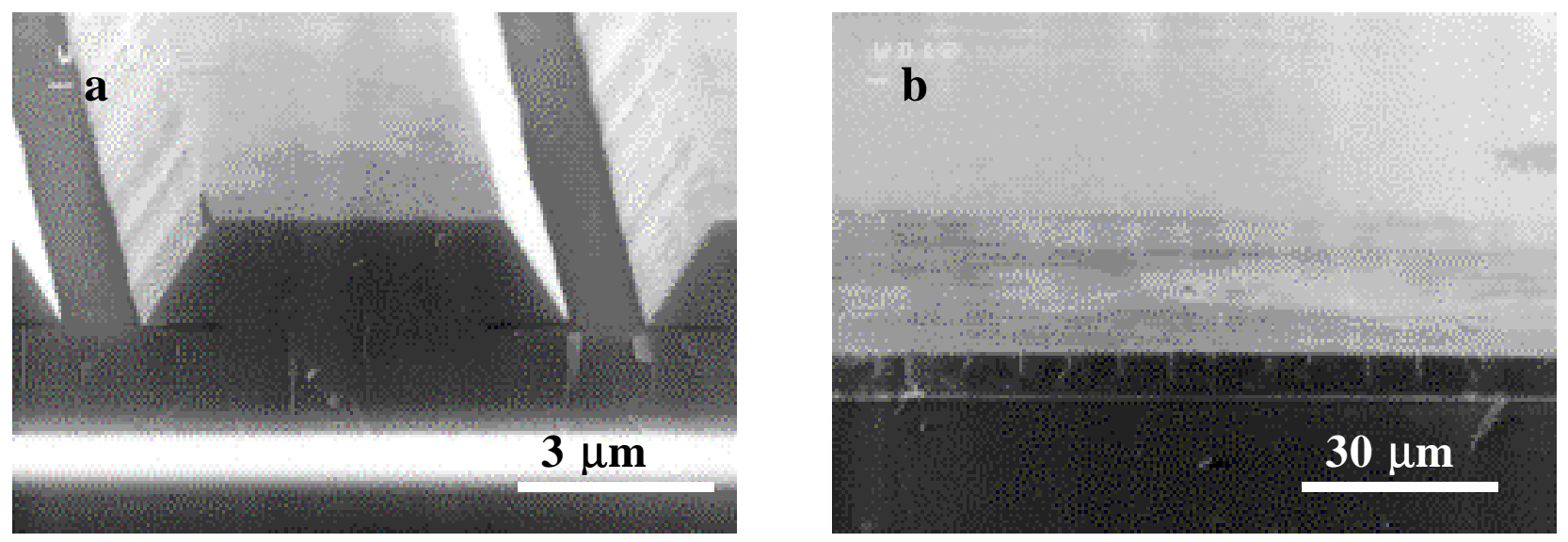

Figure $3 \mathrm{SEM}$ images of $\mathrm{GaN}$ layer grown by $\mathrm{ELO}$ on $\mathrm{SiO}_{2}$ line pattern along the $<1 \overline{1} 00>$ direction in the mixture ambient at growth times of (a) $30 \mathrm{~min}$ and (b) $120 \mathrm{~min}$. 


\section{Experimental}

A conventional atmospheric MOVPE apparatus with a horizontal reactor was used. A 3- $\mu$ m-thick undoped MOVPE-grown GaN on sapphire using a low-temperature GaN buffer layer was used as the substrate. $\mathrm{A} \mathrm{SiO}_{2}$ stripe pattern with a $4 \mu \mathrm{m}$ window width and a $4 \mu \mathrm{m}$ mask width was aligned along the $\langle 11 \overline{2} 0\rangle$ or $\langle 1 \overline{1} 00\rangle$ direction of the underlying $\mathrm{GaN}$ layer. After a 100-nm-thick $\mathrm{SiO}_{2}$ film was deposited by a radio-frequency (RF) sputtering, the $\mathrm{SiO}_{2}$ stripe pattern was fabricated by standard photolithographic processes and reactive ion etching (RIE). The growth temperature of $\mathrm{GaN}$ as measured by a thermocouple in the heating system was $1000^{\circ} \mathrm{C}$. The growth rate of $\mathrm{GaN}$ at our standard conditions was $3.5 \mu \mathrm{m} / \mathrm{hr}$. The ambient gas in the ELO process was hydrogen, nitrogen or their mixture (mixture ratio, hydrogen : nitrogen $=1: 1$ ), and was controlled by a carrier gas for metalorganic materials. However, because of using hydrogen for bubbling of metalorganic materials, small amount of hydrogen (4.2\%) was mixed with the ambient gases in all ELO processes.

The growth rates of the lateral face and the c-facet of ELO-GaN were estimated from the fieldemission scanning electron microscopy (SEM) images and the growth times.

In order to measure the dislocation density in the ELO-GaN layers, we observed the pits on an $\mathrm{In}_{0.2} \mathrm{Ga}_{0.8} \mathrm{~N}$ layer (100 nm-thick) grown on an ELO-GaN layer. The growth pit density (GPD) on the InGaN layer is considered to correspond to the dislocation density of the underlying ELO-GaN layer [14].

In order to investigate the crystallographic structure of the ELO-GaN layers, $\omega$-scan X-ray diffraction (XRD) measurements were performed on the ELO-GaN (0004) plane as a function of $\phi$ ( $\phi$ : the rotation angle of the sample about its surface normal), and reciprocal space mapping measurements were also carried out using a high-resolution X-ray diffractometer (Philips X' Pert MRD).

\section{Results and Discussions}

Figures 1(a) and (b) show SEM images of GaN on the $<1100>$ stripe pattern in hydrogen ambient and nitrogen ambient at a growth time of $30 \mathrm{~min}$, respectively. In hydrogen ambient, a (0001) facet is observed on top, and off-facets are observed on the side walls. On the other hand, the ELO-GaN grown on the $\langle 11 \overline{2} 0\rangle$ stripe pattern in hydrogen ambient had triangular cross sections with only the $\{1 \overline{1} 01\}$ facet on the side walls at the same growth time. The lateral overgrowth rate on the $\langle 1 \overline{100\rangle}$ stripe pattern was faster than that on the $\langle 11 \overline{2} 0\rangle$ stripe pattern [14]. The lateral overgrowth rate in nitrogen ambient is enhanced remarkably in contrast with that in hydrogen ambient, as shown in Figure 1(b). The ELO-GaN grown on the $\langle 11 \overline{2} 0\rangle$ stripe pattern in nitrogen ambient at the same growth time had trapezoidal cross sections with a (0001) facet on top and the $\{1 \overline{101}\}$ facet on the side walls. In nitrogen ambient, the rate of lateral overgrowth on the $\langle 1 \overline{1} 00\rangle$ stripe pattern was also faster than that on the $<11 \overline{2} 0\rangle$ stripe pattern. Nitrogen ambient improves the lateral overgrowth rate and suppresses the (0001) facet growth rate.

The fast growth rate of the (0001) facet results in the line structure of GaN with only the $\{1 \overline{1} 01\}$ facet on the side walls. The improvement of the (0001) facet in the case of nitrogen ambient indicates that the growth rate of the side walls in nitrogen ambient is slightly faster than that in hydrogen ambient. A larger amount of source materials might be consumed on the side walls in the case of nitrogen ambient. Consequently, the amount of source materials supplied to the (0001) facet is reduced, resulting in the improvement of the (0001) facet.

Figures 2(a) and (b) show SEM images of the ELO-GaN layer on the $\langle 1 \overline{1} 00\rangle$ stripe pattern in hydrogen ambient at a growth time of $120 \mathrm{~min}$, and in nitrogen ambient at a growth time of $180 \mathrm{~min}$, respectively. The ELO-GaN layer grown in hydrogen ambient is smooth, while that grown in nitrogen 
ambient is undulated, corresponding to the mask pattern. The ELO-GaN layer with a smooth surface was not obtained on the $\langle 11 \overline{2} 0\rangle$ stripe pattern for both nitrogen and hydrogen ambients.

In order to increase the lateral overgrowth rate similar to that like in nitrogen ambient and to improve surface morphology of the ELO-GaN layer similar to that in hydrogen ambient, their mixture ambient was employed for the growth. Figure 3 shows SEM images of GaN grown on the $\langle 1 \overline{1} 00\rangle$ stripe pattern at growth times of $30 \mathrm{~min}$ and $120 \mathrm{~min}$, respectively. A smooth surface was likewise obtained, similar to that in hydrogen ambient. Table 1 shows the rate of lateral overgrowth and (0001) facet in each ambient. The lateral overgrowth rate in nitrogen ambient $(4.3 \mu \mathrm{m} / \mathrm{hr})$ is faster than that in hydrogen ambient $(2.6 \mu \mathrm{m} / \mathrm{hr})$, while the (0001) facet growth rate in nitrogen ambient $(2.1 \mu \mathrm{m} / \mathrm{hr})$ is slower than that in hydrogen ambient $(5.5 \mu \mathrm{m} / \mathrm{hr})$. From this result, it is found that the lateral overgrowth rate is enhanced by using the mixture ambient.

Table1 Lateral growth rate and (0001) facet growth rate of ELO-GaN on the $<1 \overline{1} 00\rangle$ stripe pattern in hydrogen ambient, nitrogen ambient and their mixture ambient.

\begin{tabular}{|c|c|c|}
\hline & $\begin{array}{c}\text { Lateral growth rate } \\
(\mu \mathrm{m} / \mathrm{hr})\end{array}$ & $\begin{array}{c}(0001) \text { facet growth rate } \\
(\mu \mathrm{m} / \mathrm{hr})\end{array}$ \\
\hline $\mathrm{H}_{2}$ ambient & 2.6 & 5.5 \\
\hline $\mathrm{H}_{2}+\mathrm{N}_{2}$ ambient & 3.0 & 4.0 \\
\hline $\mathrm{N}_{2}$ ambient & 4.3 & 2.1 \\
\hline
\end{tabular}

Table 2 Dislocation density of ELO-GaN grown in the mixture.

\begin{tabular}{|c|c|c|}
\hline \multicolumn{2}{|c|}{ On mask } & \multirow{2}{*}{ On window } \\
\cline { 1 - 2 } ELO region & Coalescence region & \\
\hline$<10^{7} \mathrm{~cm}^{-2}$ & $6 \times 10^{7} \mathrm{~cm}^{-2}$ & $4 \times 10^{8} \mathrm{~cm}^{-2}$ \\
\hline
\end{tabular}

Figure 4 shows the distribution of pits on InGaN surface grown on the ELO-GaN in the mixture ambient, where the dots were obtained from the SEM image, and the bars indicate the positions of $\mathrm{SiO}_{2}$ masks under the ELO-GaN. The pit density can be seen in the coalescence regions on the masks and in the window regions. The density of dislocations above the masks decreased significantly, but dislocations were observed in the coalescence regions on the center of the masks. The dislocation densities obtained from the SEM image are less than $10^{7} \mathrm{~cm}^{-2}$ above the masks and $4 \times 10^{8} \mathrm{~cm}^{-2}$ above the window regions, respectively, as shown in Table 2 . These are of the same order as the dislocation density in hydrogen ambient [14].

Figure 5 shows the full-widths at half maximum (FWHMs) of the XRD rocking curves for the (0004) plane as a function of $\phi$. It is found that the FWHMs of the ELO-GaN layer are dependent on $\phi$. The maximum FWHMs are observed at $\phi=90^{\circ}$ and $\left.270^{\circ}(<11 \overline{2} 0\rangle\right)$, corresponding to the lateral overgrowth direction, and the minimum FWHMs are observed at $\phi=0^{\circ}$ and $180^{\circ}(\langle 1 \overline{1} 00\rangle)$, corresponding to the $\mathrm{SiO}_{2}$ stripe pattern. It is considered that the FWHM reflects the crystallographic tilting of the ELO-GaN layer. The FWHMs of a 4- $\mu$ m-thick undoped GaN grown by MOVPE without ELO in hydrogen ambient is not dependent on $\phi$ as shown in Figure $5\left(\right.$ Ref. $\left.\mathrm{H}_{2}\right)$. Although the ELO process using hydrogen ambient reduces the dislocation density of the GaN layer, it introduces the anisotropic crystallographic tilting. The anisotropic tilting is drastically enhanced in nitrogen ambient, but the tilt angle is smaller than that reported by Sakai et al. [15]. The mechanism of this phenomenon is under study. 


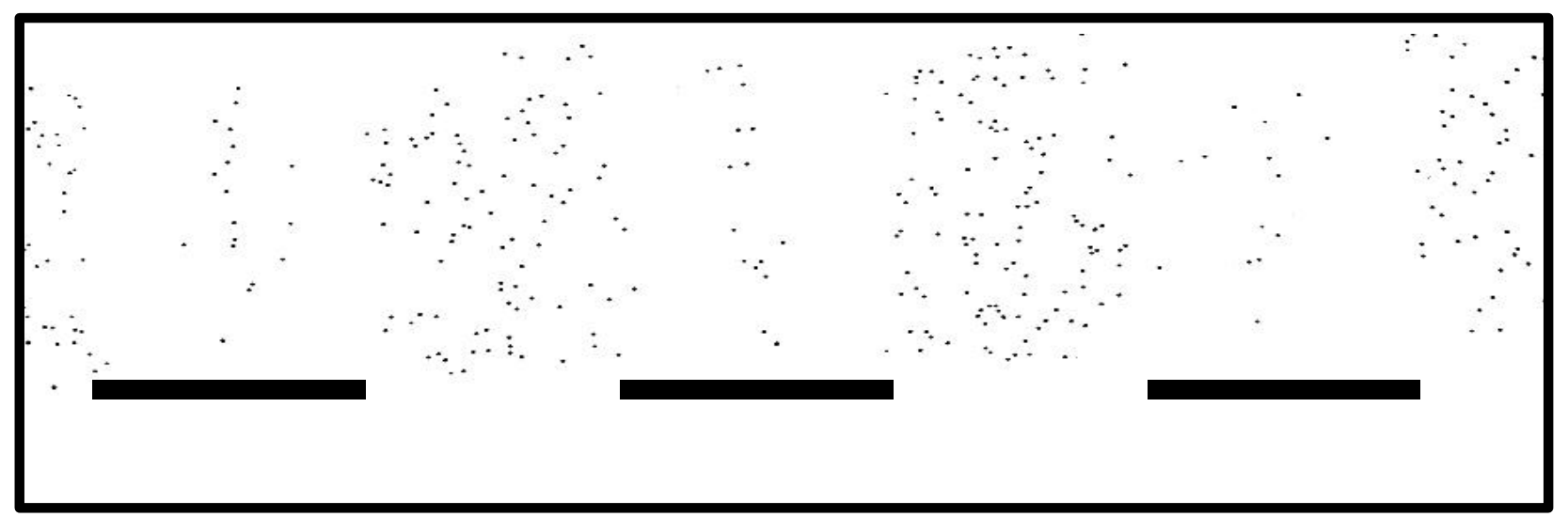

Figure 4 Distribution of pits on InGaN grown on GaN layer by ELO in the mixture ambient. Bars indicate the positions of $\mathrm{SiO}_{2}$ masks under the ELO-GaN.

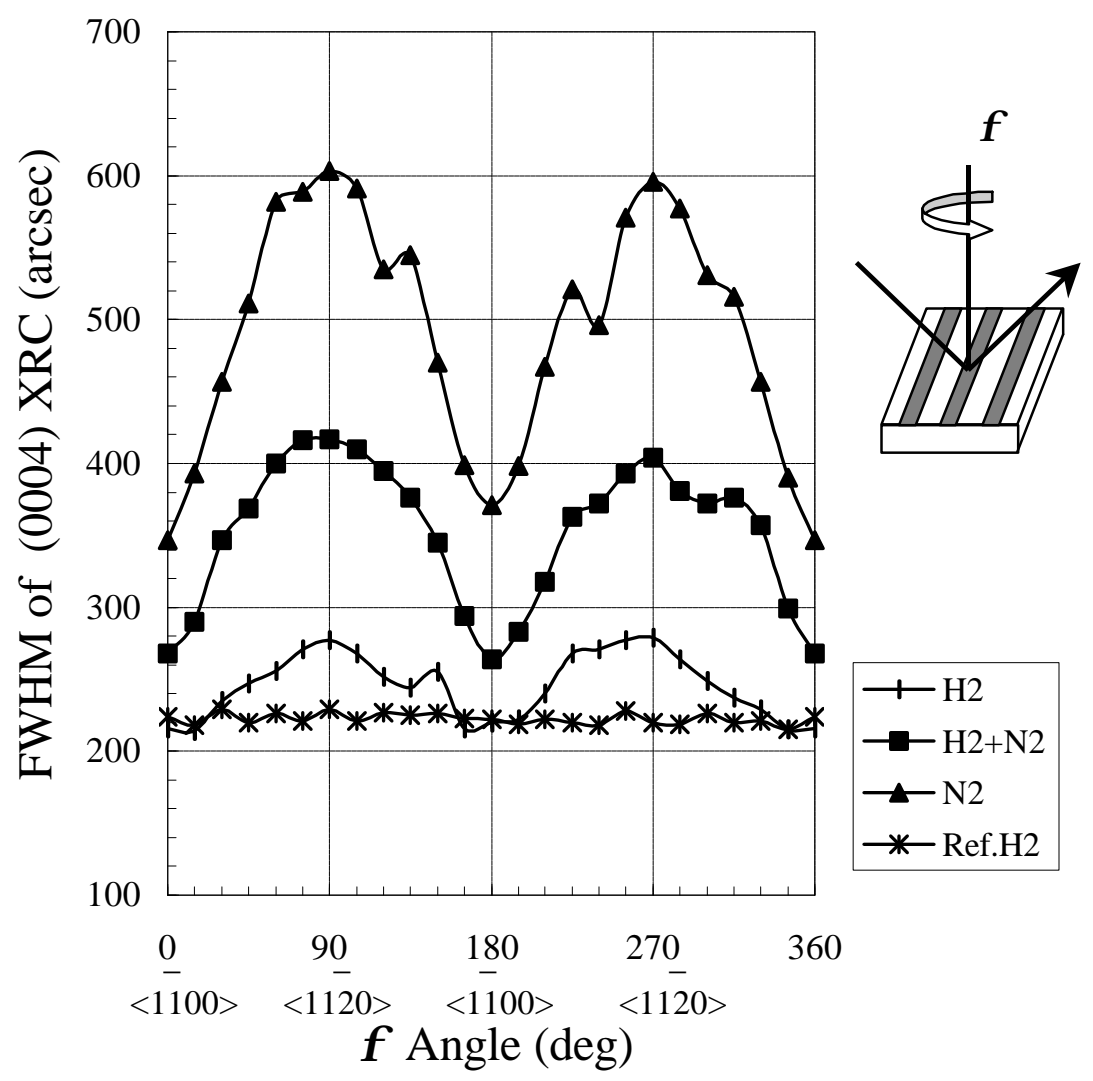

Figure 5 The FWHMs of XRD rocking curves for the (0004) GaN plane as a function of $\phi$. Ref. $\mathrm{H}_{2}$ indicates $\mathrm{GaN}$ on sapphire without ELO in hydrogen ambient. 


\section{Conclusion}

We investigated the effects of hydrogen ambient, nitrogen ambient and their mixture ambient on ELO-GaN via atmospheric MOVPE. In hydrogen ambient, the ELO-GaN with a smooth surface was obtained using the $\langle 1 \overline{1} 00\rangle$ stripe pattern, but the layer became rough on the $\langle 11 \overline{2} 0\rangle$ stripe pattern. In nitrogen ambient, lateral overgrowth rate was very fast especially on the $\langle 1 \overline{1} 00\rangle$ stripe pattern, but it was difficult to realize a smooth ELO layer surface. The mixture ambient allowed us to realize the fast lateral overgrowth rate and the smooth surface simultaneously. The reduction of the dislocation density in the ELO region was confirmed in the case of the mixture ambient. XRD analysis revealed the anisotropic crystallographic tilting of ELO-GaN toward the $\langle 11 \overline{2} 0\rangle$, which corresponds to the lateral growth direction. Nitrogen ambient enhanced the tilting of ELO-GaN.

\section{Acknowledgements}

The authors would like to acknowledge the encouragement and support of Tadao Tachibana, Kouichi Suzuki and Keisuke Imai of Mitsubishi Cable Industries, Ltd.

[1] S. Nakamura, T. Mukai and M. Senoh, Appl. Phys. Lett. 64 (1994) 1687.

[2] S. Nakamura, M. Senoh, S. Nagahama, N. Iwasa, T. Yamada, T. Matsushita, H. Kiyoku and Y. Sugimoto, Jpn. J. Appl. Phys. 35 (1996) L74.

[3] S. D. Lester, F. A. Ponce, M. G. Craford and D. A. Steigerwald, Appl. Phys. Lett. 66 (1995) 1249.

[4] S. Nakamura, M. Senoh, S. Nagahata, N. Iwasa, T. Yamada, T. Matsushita, H. Kiyoku, Y. Sugimoto, T. Kozaki, H. Umemoto, M. Sano and K. Chocho, Proc. 2nd Int. Conf. on Nitride Semicond. (Tokushima, 1997) 444.

[5] Y. Kato, S. Kitamura, K. Hiramatsu and N. Sawaki, J. Crystal Growth 144 (1994) 133.

[6] X. Li, A. M. Jones, S. D. Roh, D. A. Turnbull, E. E. Reuter, S. Q. Gu, S. G. Bishop and J. J. Coleman, Mat. Res. Soc. Symp. Proc. 395 (1996) 943.

[7] A. Usui, H. Sunakawa, A. Sakai and A. Yamaguchi, Jpn. J. Appl. Phys. 36 (1997) L899.

[8] T. S. Zheleva, O.-H. Nam, M. D. Bremser and R. F. Davis, Appl. Phys. Lett. 71 (1997) 2472.

[9] O.-H. Nam, M. D. Bremser, T. S. Zheleva and R. F. Davis, Appl. Phys. Lett. 71 (1997) 2638.

[10] D. Kapolnek, S. Keller, R. Vetury, R. D. Underwood, P. Kozodoy, S. P. DenBaars and U. K. Mishra, Appl. Phys. Lett. 71 (1997) 1204.

[11] H. Matsushima, M. Yamaguchi, K. Hiramatsu and N. Sawaki, Proc. 2nd Int. Conf. on Nitride Semicond. (Tokushima, 1997) 492.

[12] J. Park, P. A. Grudowski, C. J. Eiting and R. D. Dupuis, Appl. Phys. Lett. 73 (1998) 333.

[13] B. Beaumont, P. Gibart, M. Vaille, S. Haffouz, G. Nataf and A. Bouille, Proc. 2nd Int. Conf. on Nitride Semicond. (Tokushima, 1997) 412.

[14] K. Hiarmatsu, H. Matsushima, T. Shibata, N. Sawaki, K. Tadatomo, H. Okagawa, Y. Ohuchi, Y. Honda and T. Matsue, Mat. Res. Soc. Symp. 482 (1998) 257.

[15] A. Sakai, H. Sunakawa and A. Usui, Appl. Phys. Lett. 73 (1998) 481. 